\title{
Virtual and thermal Schwinger processes
}

\author{
Patrick Draper \\ Department of Physics, University of Illinois, Urbana, Illinois 61801, USA \\ and Amherst Center for Fundamental Interactions, Department of Physics, \\ University of Massachusetts, Amherst, Massachusetts 01003, USA
}

(Received 23 October 2018; published 27 December 2018)

\begin{abstract}
Electric flux may be screened by pair nucleation of heavy charges, a process that has a simple description in terms of a worldline instanton. When flux is wrapped around a small compact spatial dimension, worldline instantons still induce flux dissipation, but the leading process does not create real charged pairs. Instead, dissipation can be described in effective field theory as the production of long-wavelength scalar quanta via parametric resonance. The rate is computed semiclassically, and comments are made on the related problem of pair creation at finite temperature, for which differing results appear in the literature. Flux dissipation and the weak gravity conjecture together imply that the proper distance in field space a homogeneous axion field can traverse is bounded.
\end{abstract}

DOI: 10.1103/PhysRevD.98.125014

\section{INTRODUCTION}

Electric fields can be screened by the spontaneous creation of electron-positron pairs [1]. In the weak, constant-field limit, $e E \ll m^{2}$, the pair production rate can be computed semiclassically in the worldline formalism. An instanton, in this case a Euclidean circle of radius $r_{0}=m / e E$, contributes an imaginary part to the effective action for the electromagnetic field of order $e^{-\pi m r_{0}} \ll 1$. Cutting the circle at the moment of time-symmetry and analytically continuing back to Minkowski space, the classical solution describes the nucleation of the charged pair at a separation $2 r_{0}$ and their subsequent acceleration away from each other in the background field.

In this paper I revisit Schwinger pair production in the presence of a compact dimension, either a spatial or thermal circle. New semiclassical phenomena of lower action arise when the circumference of the circular dimension is less than $2 r_{0}$. The primary focus here will be on the spatial case, which is the simpler of the two; subsequently I will comment on the thermal case, which has been a source of disagreement in recent literature. For spatial circles, the lowest-action worldline instanton has winding number \pm 1 and has positive fluctuation determinant. At low energies, it generates a term in the effective action that carries an

\footnotetext{
*pdraper@illinois.edu
}

Published by the American Physical Society under the terms of the Creative Commons Attribution 4.0 International license. Further distribution of this work must maintain attribution to the author(s) and the published article's title, journal citation, and DOI. Funded by SCOAP.
Aharonov-Bohm phase. Consequently, the instanton is still responsible for flux dissipation, proceeding through longwavelength parametric resonance rather than production of heavy charged pairs.

The spatial case was previously discussed in [2]. In terms of the instantons described here, the worldline studied in [2] is an instanton-antiistanton event; it carries no phase and does not contribute to parametric resonance. Pair production at finite temperature was studied long ago in $[3,4]$, which did not rely on the semiclassical approximation and showed that the thermal correction to pair production vanishes at one loop order. In [5] it was shown that there is a finite correction at two loop order in the low temperature regime (where the thermal circle is large). Recently the thermal case has been revisited in the semiclassical approximation in the regime where the thermal circle is smaller than $2 r_{0}$. References $[2,6]$ reached different conclusions regarding the relevant semiclassical trajectories, and the problem was analyzed further in [7-9]. References $[2,7,8]$ reach similar conclusions and in particular [7] performs a numerical study of the fluctuation spectrum around their instantons, showing the presence of the required negative mode. I will argue that the trajectories considered by [6] in the thermal case are relevant to the spatial circle, and attempt to clarify the nature of the negative fluctuation mode around the instantons of $[2,7]$.

In the spatial case, from the low energy point of view, the parametric resonance process describes the fragmentation of an axion field moving coherently with some velocity. Imposing the weak gravity conjecture on the mass of the charged matter, a bound is obtained on the maximum distance the axion can travel in field space. 


\section{WORLDLINE INSTANTON AMPLITUDE}

We consider electrodynamics on $\mathbb{R}^{4} \times \mathbb{S}^{1}$ with circle circumference $L$ and a charged scalar of mass $m \gg 1 / L$, and focus on the terms in the Euler-Heisenberg action sensitive to $L$. Weak coupling is assumed for simplicity, $e \ll 1$, and only constant electric flux in the compact dimension is turned on. I will also comment on the $1+1$ dimensional case below.

Integrating out the matter field, the Euclidean effective action is

$$
\mathcal{S}_{\text {eff }}=\mathcal{S}_{0}-Z_{1}
$$

where $\mathcal{S}_{0}$ is the classical action, and the trace log correction can be expressed as a partition function of closed loops, ${ }^{1}$

$$
\begin{aligned}
Z_{1} & =-\operatorname{Tr} \log \left(-D^{2}+m^{2}\right) \\
& =\int_{0}^{\infty} \frac{d \beta}{\beta} e^{-\beta m / 2} \int \mathcal{D} x e^{-\mathcal{A}}, \\
\mathcal{A} & =\int_{0}^{\beta} d \lambda\left(\frac{1}{2} m\left(\partial_{\lambda} x^{M} \partial_{\lambda} x_{M}\right)-i e A_{M} \partial_{\lambda} x^{M}\right) \\
& =\int_{0}^{\beta} d \lambda\left(\frac{1}{2} m\left(\partial_{\lambda} x^{M} \partial_{\lambda} x_{M}\right)+e E_{5} x^{5} \partial_{\lambda} \tau\right)-\left.i e A_{5} x^{5}(\lambda)\right|_{0} ^{\beta}
\end{aligned}
$$

Here $\tau=i t$ is the Euclidean time and $x_{5} \sim x_{5}+L$ is the circle coordinate. In the last line, $A^{0}=0$ gauge is taken, and $A_{5}=A_{5}(\tau)$ with field strength $E_{5}=i \partial_{\tau} A_{5}$.

The path integral over $x$ can be computed exactly for constant $E_{5}$ by expanding around classical solutions satisfying periodic boundary conditions. The leading $L$ dependent terms are obtained from solutions for which $x^{5}(\beta)-x^{5}(0)= \pm L$. The equations of motion are

$$
\begin{aligned}
& -m \partial_{\lambda}^{2} x_{5}+e E_{5} \partial_{\lambda} \tau=0 \\
& -m \partial_{\lambda}^{2} \tau-e E_{5} \partial_{\lambda} x_{5}=0
\end{aligned}
$$

The solutions are arcs of circles of angular size $\int d \theta= \pm \beta / 2 r_{0}$. Examples are shown in Fig. 1. For angular lengths of magnitude greater than $2 \pi$, the orbits appear as closed circles but end at $x^{5}(\beta)=x^{5}(0) \pm L$, indicating winding number \pm 1 around the fifth dimension. finite,

The instantons are singular, but the classical action is

$\mathcal{A}_{0}=\frac{1}{4} e E_{5} L^{2} \cot (\omega \beta), \quad \omega=1 / 2 r_{0}=e E_{5} / 2 m$.

Since the action is quadratic, the fluctuations are insensitive to the classical solution,

\footnotetext{
${ }^{1}$ For a textbook treatment, see $[10]$.
}

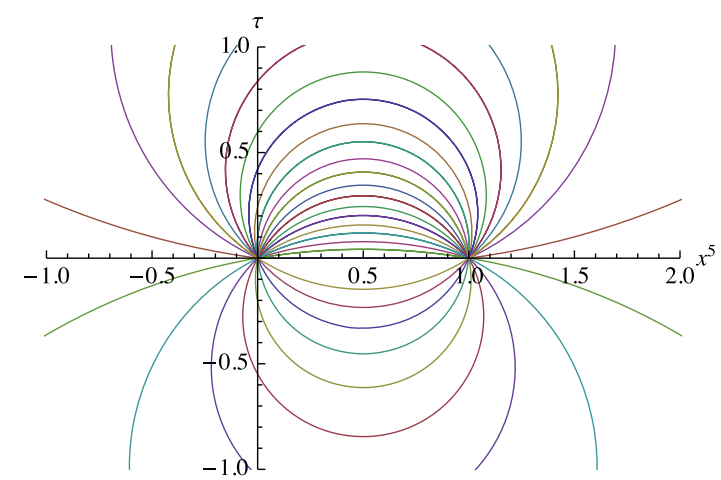

FIG. 1. Example Euclidean solutions to Eq. (4) for different values of $\beta\left(L=1, r_{0}=1, x^{5}(\beta)=x^{5}(0)+L\right)$.

$$
\mathcal{A}(\delta x)=\int_{0}^{\beta}\left[\frac{1}{2} m\left(\partial_{\lambda} \delta x_{M} \partial_{\lambda} \delta x^{M}\right)+e E_{5} \delta x^{5} \partial_{\lambda} \delta \tau\right] .
$$

The determinant is then the same as for solutions with zero winding number,

$$
\left(\operatorname{det} \mathcal{O}_{\text {free }}\right)^{-3 / 2}\left(\operatorname{det} \mathcal{O}_{\tau x^{5}}\right)^{-1 / 2}
$$

with three powers of the free 1D particle partition function

$$
\left(\operatorname{det} \mathcal{O}_{\text {free }}\right)^{-1 / 2} \rightarrow V_{1} \sqrt{\frac{m}{2 \pi \beta}}
$$

and

$$
\begin{aligned}
\operatorname{det} \mathcal{O}_{\tau x^{5}} & =\left(\operatorname{det} \mathcal{O}_{\text {free }}\right)^{2} \prod_{n \neq 0} \operatorname{det}\left[\begin{array}{cc}
1 & \left(\frac{i \omega \beta}{\pi n}\right) \\
-\left(\frac{i \omega \beta}{\pi n}\right) & 1
\end{array}\right] \\
& =\left(\operatorname{det} \mathcal{O}_{\text {free }}\right)^{2} \prod_{n \neq 0}\left[1-\left(\frac{\omega \beta}{\pi n}\right)^{2}\right] \\
& =\left(\operatorname{det} \mathcal{O}_{\text {free }}\right)^{2}\left(\frac{\sin (\omega \beta)}{\omega \beta}\right)^{2}
\end{aligned}
$$

The leading $L$-dependent contribution to $Z_{1}$ is therefore

$$
\begin{aligned}
Z_{1}^{L}= & -2 V_{5} \int_{0}^{\infty} \frac{d \beta}{\beta}\left(\frac{\omega \beta}{\sin (\omega \beta)}\right)\left(\frac{m}{2 \pi \beta}\right)^{5 / 2} \\
& \times e^{-\frac{1}{2} m\left(\beta+\omega L^{2} \cot [\omega \beta]\right)} \cos \left(A_{5} L e\right)
\end{aligned}
$$

with $\cos \left(A_{5}\right)$ dependence arising from the boundary term in Eq. (3) and the sum over $\Delta x^{5}= \pm L$.

The contour of integration for $\beta$ must be chosen to avoid the singularities at $\beta=\pi n / \omega$. For large $m$ the integral can then be evaluated semiclassically. For small circles and weak fields, $\omega L=L / 2 r_{0}<1$, the leading stationary point is real, 


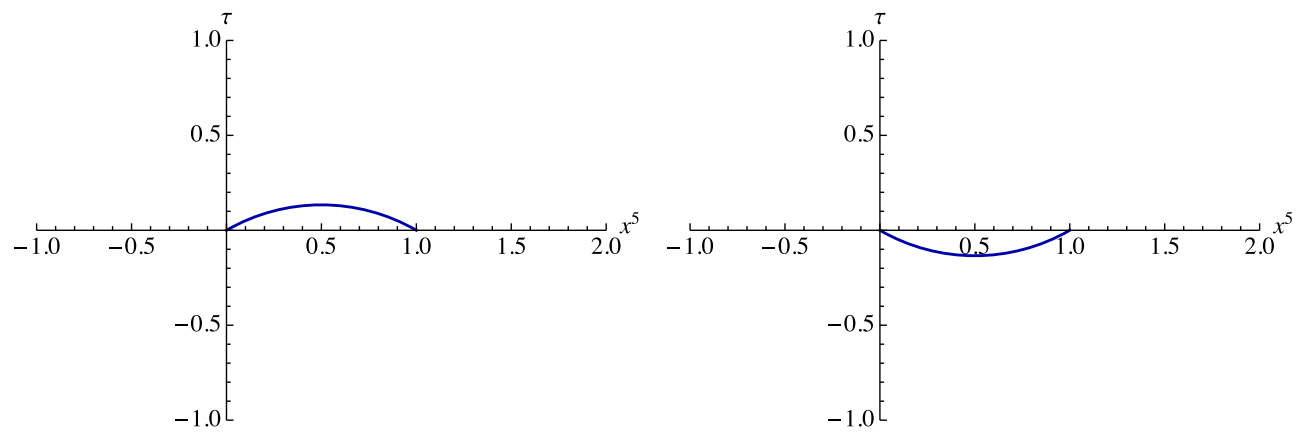

FIG. 2. Instanton with $\beta=\beta_{0}$. Parameters the same as in Fig. 1. Right: anti-instanton.

$$
\beta_{0}=\frac{1}{\omega} \sin ^{-1}(\omega L)
$$

and its contribution to the integral is free from ambiguities associated with the initial choice of contour. Figure 2 shows the instanton with $\beta=\beta_{0}$ taken from the family plotted in Fig. 1. We obtain

$$
\begin{aligned}
Z_{1}^{L} / V^{5} & =-B e^{-\frac{1}{2} m L\left(\sqrt{1-\omega^{2} L^{2}}+\omega^{-1} L^{-1} \sin ^{-1}(\omega L)\right)} \cos \left(A_{5} L e\right) \\
B & =\frac{m^{2} \omega^{5 / 2}}{2 \pi^{2} L^{1 / 2}\left(1-\omega^{2} L^{2}\right)^{1 / 4}\left(\sin ^{-1}(\omega L)\right)^{5 / 2}} .
\end{aligned}
$$

In the $E \rightarrow 0$ limit, this result must reproduce the leading term in the Wilson line effective potential for the background $A_{5}$, for which many exact (non-semiclassical) computations exist in the literature [11-16]. Expanding Eq. (12) for $\omega L \ll 1$ we obtain

$$
\begin{aligned}
Z_{1}^{L} / V^{5}= & -\frac{m^{2}}{2 \pi^{2} L^{3}} e^{-m L\left(1-\frac{1}{4} L^{2} \omega^{2}+\mathcal{O}\left(\omega^{4} L^{4}\right)\right)} \\
& \times\left(1+\frac{1}{4} L^{2} \omega^{2}+\mathcal{O}\left(\omega^{4} L^{4}\right)\right) \cos \left(A_{5} L e\right) .
\end{aligned}
$$

For $\omega=0$, Eq. (13) coincides with the leading term in the effective potential on $R^{4} \times S^{1}$ generated by a heavy complex scalar field of charge one, $V\left(A_{5}\right)=-\frac{m^{2}}{2 \pi^{2} L^{3}} e^{-m L} \cos \left(A_{5} L e\right)$ for $m L \gg 1$. Turning on a finite background electric field reduces the exponential suppression of the amplitude. It should also be possible to obtain a generalization of Eq. (12) by non-semiclassical methods. For example, in [17], Diakonov and Oswald compute the contribution of massless charged fermions to the effective action at finite temperature allowing for background electric and magnetic fields. The massless limit is complementary to the semiclassical limit of large $m$, and it would be of interest to have a result valid in all regimes, although we do not pursue this calculation further here.

Furthermore, for $\omega L<1$, the instanton contribution is real: the leading semiclassical effect does not directly describe pair production or other decay modes for the flux. It describes a virtual process before and after which the flux has the same value. Nonetheless, the amplitude is connected with a rather different type of flux-reducing process occurring in the long-distance theory, when the instanton amplitude is suitably interpreted as a Wilsonian effective action.

In addition to $\beta_{0}$, Eq. (13) exhibits infinitely many saddle points of higher action. The second saddle above the one shown in Fig. 2 is given in Fig. 3. This instanton (and all subsequent even-numbered saddles) has a negative fluctuation eigenvalue corresponding to fluctuations in the $\beta$ direction, yielding an imaginary contribution to the effective action. The real part of the action is of order $\pi m r_{0}$ for small $\omega L$, associated with real pair production.

\section{III. $S_{\text {eff }}$ AND PARAMETRIC RESONANCE}

It is convenient to work at distances larger than $L$, where $\mathcal{S}_{\text {eff }}$ becomes a Wilsonian action for the four-dimensional gauge field and the periodic scalar zero mode of $A_{5}$, obtained by the substitutions

$$
\begin{aligned}
A_{5} L e & \rightarrow \phi / f(f=1 / e L) \quad E_{5} \rightarrow E_{5} \\
\phi & \rightarrow E_{5} t+\varphi(x, t) .
\end{aligned}
$$

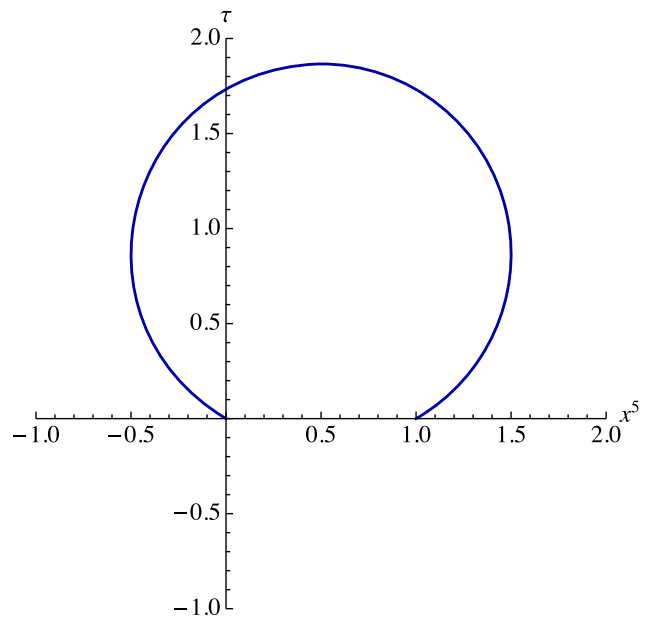

FIG. 3. Instanton with $\beta=\beta_{1}$, the saddle point of the secondlowest action in Eq. (13) and the first saddle with negative fluctuations. Parameters the same as in Fig. 1. 
Here $E_{5}$ is a constant background velocity for $\phi$, and $\phi$ is referred to as an axion. The meaning of the second line of Eq. (14) is that $E_{5}$ dependence already present in Eq. (13) through $\omega$ remains simply $E_{5}$, without introducing any $\dot{\varphi}$ terms. This truncation is necessary for the consistency of the derivative expansion. Furthermore, we will see shortly that we are interested in Fourier modes of $\varphi$ with momenta of order $e E_{5} L$. Consistently retaining the background $E_{5}=$ $\dot{A}_{5}$ without computing $\partial_{\mu} \varphi$ terms in the effective action requires $e E_{5} L \varphi \lesssim E_{5} \Rightarrow \varphi \lesssim f$. In other words, we must expand in small fluctuations $\varphi / f$.

Thus, at leading order in the derivative and semiclassical expansions and to quadratic order in $\varphi$, the scalar effective Lagrangian is

$$
\begin{aligned}
& \mathcal{L}_{\text {eff }, 4 d}(\varphi) \\
& \quad=\frac{1}{2}(\partial \varphi)^{2}-\frac{m^{2}}{2 \pi^{2} L^{2}}\left(\epsilon \frac{\varphi}{f} \sin (\Omega t)-\frac{1}{2}\left(\frac{\varphi}{f}\right)^{2} \epsilon \cos (\Omega t)\right),
\end{aligned}
$$

where

$$
\epsilon \equiv e^{-m L\left(1-\frac{1}{4} L^{2} \omega^{2}\right)}, \quad \Omega \equiv e E_{5} L
$$

retaining only the leading term in $\omega L$ in the exponent.

The quadratic term in $\varphi$ captures the leading effect responsible for the dissipation of flux in the effective theory. The linear term corrects the classical motion of the homogeneous mode; it is not important for our purposes and will subsequently be neglected. Higher-order EulerHeisenberg terms are not exponentially-suppressed, but can also be omitted because they are flux-conserving. Real pair creation of the original charged fields cannot be described in the EFT, but has a much larger $\mathcal{O}\left(m r_{0} \gg m L\right)$ classical action and is therefore exponentially subdominant. ${ }^{2}$

In backgrounds of constant $E_{5}$, the mass term in (15) is time-dependent, leading to a parametric resonance instability [18]. Amplitudes for modes of the axion with wave vectors $|k| \propto E_{5}$ grow exponentially in time. The rate of the energy transfer to these modes may be obtained in several different ways. For nonzero $k$, the fluctuation equation of motion is

$$
\ddot{\varphi}(k, t)+k^{2} \varphi(k, t)+\epsilon \Lambda^{2} \cos (\Omega t) \varphi(k, t)=0
$$

\footnotetext{
${ }^{2}$ We also note that it was not necessary to completely dimensionally reduce. The reduction simplifies the analysis and is sufficient for fields weak compared to the compactification scale, $e E_{5} L^{2}<1$, but in principle it could be extended to include additional modes. Kaluza-Klein modes of $A_{5}$ up to level $n \sim m L$ could be retained in the effective theory below $m$, and modes up to $n \sim e E_{5} L^{2}$ could play a role in the following discussion of dissipation.
}

where the scale $\Lambda^{2} \equiv \frac{m^{2}}{2 \pi^{2} f^{2} L^{2}}=\frac{e^{2} m^{2}}{2 \pi^{2}}$ is introduced to simplify notation. The $\epsilon$ term is a perturbation. Its first-order effect is to mix waves of frequency $\omega_{k}= \pm|k|$ with waves of frequency $\omega_{k} \pm \Omega$. For generic $|k|$, the mixing is small and the solutions are stable. However, for $|k| \approx \Omega / 2$, degenerate positive and negative frequency modes $\omega_{k} \approx$ $\pm \Omega / 2$ mix with each other, and the mixing has a more substantial effect. To analyze this case, we can restrict our attention to deviations of order $\epsilon$ around $|k|=\Omega / 2$,

$$
k^{2} \rightarrow(\Omega / 2+\epsilon \delta k)^{2}
$$

and take the ansatz

$$
\begin{aligned}
\varphi \rightarrow & e^{i\left(\Omega / 2+\epsilon \delta \omega_{k}\right) t}+c_{0} e^{i\left(-\Omega / 2+\epsilon \delta \omega_{k}\right) t} \\
& +\epsilon c_{+} e^{3 i \Omega t / 2}+\epsilon c_{-} e^{-3 i \Omega t / 2} .
\end{aligned}
$$

This ansatz allows the waves with zeroth-order frequencies $\omega_{k}= \pm \Omega / 2$ to mix with each other at zeroth order, and with modes of frequency $\pm 3 \Omega / 2$ at first order. The equation of motion can then be solved to $\mathcal{O}(\epsilon)$ for the coefficients $c_{0,+,-}$ and the frequency shift. The result for the frequency shift is

$$
\epsilon \delta \omega_{k}= \pm \frac{\epsilon}{2 \Omega} \sqrt{-\Lambda^{4}+4 \Omega^{2} \delta k^{2}} .
$$

In the band $|\epsilon \delta k|<\epsilon \Lambda^{2} / 2 \Omega, \delta \omega_{k}$ is imaginary, and some of the modes grow exponentially with $t$. For example, in the center of the band $(\delta k=0)$, the mode grows as

$$
\varphi \sim e^{i \Omega t / 2+\epsilon\left(\Lambda^{2} / 2 \Omega\right) t} .
$$

Consequently, the energy in the first band grows approximately as

$$
\begin{aligned}
E_{\text {first band }} & \sim \omega_{k}\left\langle|\varphi(k, t)|^{2}\right\rangle d^{3} k \\
& \sim(\Omega)^{2}\left(\epsilon \frac{\Lambda^{2}}{\Omega}\right)\left\langle|\varphi(k, 0)|^{2}\right\rangle e^{\epsilon\left(\Lambda^{2} / \Omega\right) t} .
\end{aligned}
$$

At higher orders in $\epsilon$, instabilities appear in frequency bands centered on every $n \Omega / 2$.

These results have a simple general description. In the absence of charged matter, electric flux is conserved. Electric fields along a compact direction are constant as a result of improper gauge transformations, a global shift symmetry acting as $A_{5} \rightarrow A_{5}+c / L, c \sim c+2 \pi$. Charged matter breaks the global symmetry, so some flux nonconserving processes are expected to survive in a longdistance effective theory. The leading process is parametric resonance, driven by the ordinary Wilson line effective potential terms $\sim e^{-m L} \cos \left(n A_{5}\right)$. This result could be obtained without the detailed semiclassical calculation of the previous section; however, the worldline instanton 
captures electric-flux-dependent corrections to the exponent that reduce the suppression of the rate. This reduction was also noted in [2], although the instanton and antiinstanton in Fig. 2 were treated as a single event, and so the result did not contain the harmonic terms in $A_{5}$ that are critical to flux dissipation. It was also suggested in [2] that the instanton describes annihilation of virtual electrons into photons, a process which is not captured by the leading semiclassical approximation. Instead, dissipation arises in the EFT by production of scalar quanta.

The discussion above is relevant in $d>1$ spatial dimensions. The $1+1$ dimensional case is somewhat different, but also of interest. Here the electric field acts as a $\theta$-term [19], and Schwinger pair production describes tunneling between different metastable branches, analogous to phenomena arising in various $4 \mathrm{~d}$ gauge theories [20]. Dissipation by parametric resonance, however, does not occur when space is compactified on a small circle. In this case the effective theory at distances long compared to $L$ is the quantum mechanics of a 1D rotor $\phi(t)$. Flux/ angular momentum $\sim \dot{\phi}$ is quantized and conserved at $\mathcal{O}\left(\epsilon^{0}\right)$. The contribution to the Hamiltonian at $\mathcal{O}(\epsilon)$ does not commute with the angular momentum and describes mixing of different flux states.

\section{FINITE TEMPERATURE}

The instantons studied above in the case of a spatial dimension have also been suggested to be relevant at finite temperature [6]. Here I will comment briefly on the relation of the finite temperature and spatial circle instantons, without attempting a complete discussion of the thermal problem.

Schwinger pair production at finite temperature was studied in $3+1$ dimensions in $[3,4]$, where it was found that corrections vanish at one loop order. Subsequently it was shown in [5] that the two loop correction is nonvanishing. These analyses do not rely on the semiclassical approximation. However, the analysis of [5] holds for low temperatures, and does not apply when the thermal circle is smaller than twice the Schwinger radius $r_{0}$. Recently a number of works have studied this regime semiclassically [2,6-9]. In particular, [6,9] analyze trajectories very similar to those studied above in the spatial case, related by a simple exchange of $x_{5}$ and $\tau$ coordinates.

Locally, arcs of the critical circle still solve the Euclidean equations of motion, regardless of the boundary conditions. However, with thermal boundary conditions $\tau \sim \tau+L$ and electric flux pointing in a noncompact spatial direction $z$, trajectories of nonzero winding number are no longer stationary points of the Euclidean action. This includes worldlines analogous to Figs. 2 and 3, swapping $x_{5} \rightarrow \tau$, $\tau \rightarrow z$, as studied in [6,9]. The $\beta$ action is a linear function of a collective coordinate $z_{0}$ describing the starting and ending point of the worldline in the noncompact flux direction. For example, the action of a trajectory analogous to Fig. 2 would be

$$
\mathcal{A}_{0}=e E_{z} L z_{0}+\frac{1}{4} e E_{z} L^{2} \cot (\omega \beta)
$$

and is not stationary under variation of $z_{0}$.

In [2], it was argued that a worldline corresponding to the fusing of the instanton and anti-instanton in Fig. 2 describes thermally-assisted pair creation. It was shown that this "lemon instanton" trajectory reproduces the exponential suppression obtained by minimizing the sum of Boltzmann and Wentzel-Kramers-Brillouin tunneling exponents, treating the tunneling process as a relativistic barrier penetration problem with linear potential. A detailed analytical and numerical study of the problem was undertaken in $3+1$ dimensions in $[7,8]$, including the role of short-distance interactions in modifying the worldline. Reference [7] found that the lemon instanton is indeed relevant in $3+$ 1 dimensions, arising as a weak coupling limit of their instantons when the thermal circle is smaller than twice the Schwinger radius.

Reference [7] also studied the low-lying fluctuation spectrum around their worldline instantons, showing that the spectrum exhibits a negative mode required in order for the solutions to have relevance to pair creation. The properties of this mode were described in the high temperature limit where the transition is entirely thermal: here the instanton is two straight lines wrapping the thermal circle, and the negative mode is a fluctuation in the separation of these lines. It is natural to ask what the negative mode is in the finite temperature, small coupling limit, where the lemon instanton provides a good approximation. Moreover, it should be easiest to understand in the $1+1$ dimensional case, where the electromagnetic field is nonpropagating.

The lemon instanton can also be described as (the boundary of) the overlap region between two circles of Schwinger radius $r_{0}$, separated by a distance $d=d_{L}$ so that the lemon "just fits" inside the thermal circle,

$$
d_{L} / 2 \equiv \sqrt{r_{0}^{2}-L^{2} / 4}
$$

At first sight, the trajectory appears puzzling: small variations of $d$ change the action at leading order in the variation. The only stationary point of the $\mathcal{O}(e)$ action appears to be $d=0$, where the circles degenerate. At higher order in $e$, different parts of the worldlines can interact; furthermore, with a compact direction, trajectories larger than $L$ begin to overlap, increasing the effects of interactions. However, it is unclear whether these effects are important in $1+1$ dimensions, where the theory is otherwise simplest, or in an extreme weak-coupling limit in higher dimensions.

Another feature of the lemon is that it marks a singular point in configuration space where continuous trajectories, for example lemons of various $d$, can first split into 


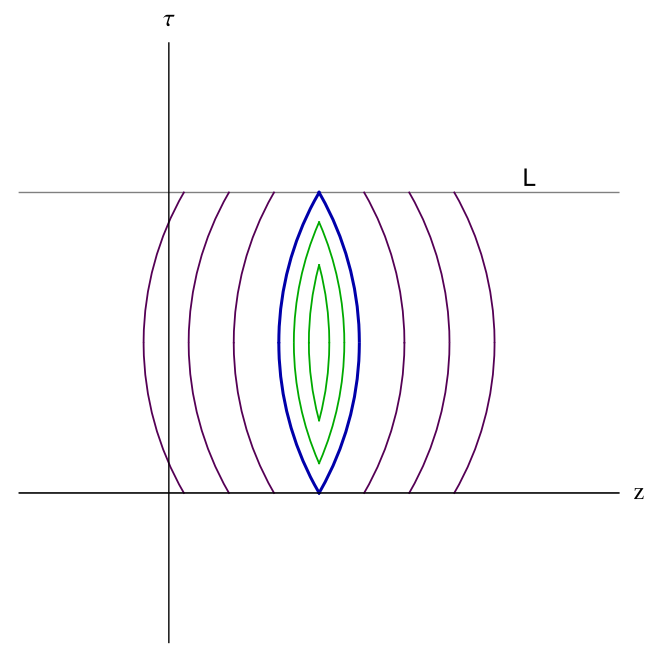

FIG. 4. A family of trajectories exhibiting the distinguished nature of the critical lemon worldline (blue). Smaller lemons (green) locally solve the equations of motion, but they are not true stationary points because the action rises monotonically in the direction of the critical lemon. Once the lemon grows to the critical size, its two sides can split apart, opening a new direction in configuration space. In this direction the action changes linearly with separation. These features suggest the presence of a saddle point with a negative mode near the critical lemon.

discontinuous pairs of opposite winding number. A family of configurations exhibiting this property is sketched in Fig. 4; among them, the critical lemon with $d=d_{L}$ maximizes the action. Smaller lemons (larger $d$ ) cannot split; this direction in field space opens up sharply when $d \rightarrow d_{L}$, allowing the action to lower again. This picture suggests that in $1+1$ dimensions the lemon is indeed a distinguished trajectory, and may be near to a genuine saddle point with a negative fluctuation eigenvalue once singularities are smoothed out by ultraviolet effects. Just as some of the solutions obtained in [7] were found to approach the lemon instanton in the weak-coupling limit, it would be interesting to examine whether the negative fluctuation modes approach Fig. 4 in the same limit.

\section{BOUNDS ON FIELD EXCURSIONS}

Theories of light axions may be subject to theoretical constraints including the weak gravity and swampland conjectures [21-26], and perhaps related to these constraints, there can be limitations on physically allowed objects and dynamical processes, particularly involving large excursions of the scalar in spacetime [27]. For example, large stationary excursions can collapse into black holes [28] or destabilize Kaluza-Klein spacetimes [29], while large axion excursions around cosmic strings cause them to inflate [30-32]. In this light we can ask whether parametric resonance places limitations on axion excursions, when the axion arises from a higher-dimensional $\mathrm{U}(1)$.

An axion with action (15) and decay constant $f$, moving with initial velocity $\dot{\phi}$, fragments into inhomogeneous modes in a timescale of order

$$
\log (t) \sim \log (1 / \epsilon) \sim m L=m / e f .
$$

In comparison, a scalar with zero potential but subject to Hubble friction decelerates as $\ddot{\phi} \lesssim-\dot{\phi}^{2} / M_{p}$, saturated when the scalar is the dominant source of energy. In a time $t$ it moves a proper distance $\Delta \phi \lesssim M_{p} \log (t)$ in field space. Inserting (25), we can estimate that the slowly fragmenting scalar can move a distance of order

$$
\Delta \phi \lesssim \frac{M_{p} m}{e f}
$$

before dissipating. Imposing a weak-gravity relation $m / e<M_{p}$, we obtain

$$
\Delta \phi \lesssim \frac{M_{p}^{2}}{f}
$$

Weak gravity also requires $f<M_{p}$, so no two distinct points in the axion field space are separated by a transPlanckian distance. But we see that the bound (27) carries additional information: even with sub-Planckian field range, the distance physically traversable by a homogeneous field is bounded.

\section{ACKNOWLEDGMENTS}

I thank L. Sorbo, A. Nahum, and G. Dunne for discussions. This work was supported by NSF Grant No. PHY-1719642.
[1] J. S. Schwinger, On gauge invariance and vacuum polarization, Phys. Rev. 82, 664 (1951).

[2] A. R. Brown, Schwinger pair production at nonzero temperatures or in compact directions, Phys. Rev. D 98, 036008 (2018).
[3] P. Elmfors and B.-S. Skagerstam, Electromagnetic fields in a thermal background, Phys. Lett. B 348, 141 (1995); Erratum, Phys. Lett. B376, 330(E) (1996).

[4] H. Gies, QED effective action at finite temperature, Phys. Rev. D 60, 105002 (1999). 
[5] H. Gies, QED effective action at finite temperature: Two loop dominance, Phys. Rev. D 61, 085021 (2000).

[6] L. Medina and M. C. Ogilvie, Schwinger pair production at finite temperature, Phys. Rev. D 95, 056006 (2017).

[7] O. Gould and A. Rajantie, Thermal Schwinger pair production at arbitrary coupling, Phys. Rev. D 96, 076002 (2017).

[8] O. Gould, A. Rajantie, and C. Xie, Worldline sphaleron for thermal Schwinger pair production, Phys. Rev. D 98, 056022 (2018).

[9] M. Korwar and A. M. Thalapillil, Finite temperature Schwinger pair production in coexistent electric and magnetic fields, Phys. Rev. D 98, 076016 (2018).

[10] H. Kleinert, Path Integrals in Quantum Mechanics, Statistics, Polymer Physics, and Financial Markets (World Scientific, 2009).

[11] Y. Hosotani, Dynamical mass generation by compact extra dimensions, Phys. Lett. 126B, 309 (1983).

[12] H. Hatanaka, T. Inami, and C. S. Lim, The gauge hierarchy problem and higher dimensional gauge theories, Mod. Phys. Lett. A 13, 2601 (1998).

[13] I. Antoniadis, K. Benakli, and M. Quiros, Finite Higgs mass without supersymmetry, New J. Phys. 3, 20 (2001).

[14] G. von Gersdorff, N. Irges, and M. Quiros, Bulk and brane radiative effects in gauge theories on orbifolds, Nucl. Phys. B635, 127 (2002).

[15] H.-C. Cheng, K. T. Matchev, and M. Schmaltz, Radiative corrections to Kaluza-Klein masses, Phys. Rev. D 66, 036005 (2002).

[16] N. Arkani-Hamed, S. Dubovsky, A. Nicolis, and G. Villadoro, Quantum horizons of the Standard Model landscape, J. High Energy Phys. 06 (2007) 078.

[17] D. Diakonov and M. Oswald, Covariant derivative expansion of fermionic effective action at high temperatures, Phys. Rev. D 70, 016006 (2004).
[18] J. H. Traschen and R. H. Brandenberger, Particle production during out-of-equilibrium phase transitions, Phys. Rev. D 42, 2491 (1990).

[19] S. R. Coleman, More about the massive Schwinger model, Ann. Phys. (N.Y.) 101, 239 (1976).

[20] E. Witten, Large N chiral dynamics, Ann. Phys. (N.Y.) 128, 363 (1980).

[21] C. Vafa, The string landscape and the swampland, arXiv: hep-th/0509212.

[22] H. Ooguri and C. Vafa, On the geometry of the string landscape and the swampland, Nucl. Phys. B766, 21 (2007).

[23] T. Banks, M. Dine, P. J. Fox, and E. Gorbatov, On the possibility of large axion decay constants, J. Cosmol. Astropart. Phys. 06 (2003) 001.

[24] N. Arkani-Hamed, L. Motl, A. Nicolis, and C. Vafa, The string landscape, black holes and gravity as the weakest force, J. High Energy Phys. 06 (2007) 060.

[25] G. Obied, H. Ooguri, L. Spodyneiko, and C. Vafa, De Sitter space and the swampland, arXiv:1806.08362.

[26] P. Agrawal, G. Obied, P. J. Steinhardt, and C. Vafa, On the cosmological implications of the string swampland, Phys. Lett. B 784, 271 (2018).

[27] T. Banks, Strings in a landscape, in String Theory: From Gauge Interactions to Cosmology (Springer, Netherlands, 2004), pp. 3-7.

[28] A. Nicolis, On super-Planckian fields at sub-Planckian energies, J. High Energy Phys. 07 (2008) 023.

[29] P. Draper and S. Farkas (to be published).

[30] A. Vilenkin, Topological Inflation, Phys. Rev. Lett. 72, 3137 (1994).

[31] M. J. Dolan, P. Draper, J. Kozaczuk, and H. Patel, Transplanckian censorship and global cosmic strings, J. High Energy Phys. 04 (2017) 133.

[32] A. Hebecker, P. Henkenjohann, and L. T. Witkowski, What is the magnetic weak gravity conjecture for axions?, Fortschr. Phys. 65, 1700011 (2017). 